\title{
High Gain Dual-Band Millimeter wave Antenna Using Flexible PET Substrate
}

\author{
Ali Ghavidel ${ }^{1,2}$, Ali Araghi $^{2}$, Sami Myllymäki ${ }^{3}$, Marko Sonkki ${ }^{4}$ \\ ${ }^{1}$ Iran National Elite Foundation (INEF), ${ }^{2}$ Iran Telecommunication Research Center (ITRC), Tehran, Iran, \\ \{a.ghavidel, a.araghi\}@itrc.ac.ir, \\ ${ }^{3}$ Microelectronics Research Unit (MIC), University of Oulu, Oulu, Finland, sami.myllymaki@ oulu.fi \\ ${ }^{4}$ Centre for Wireless Communication (CWC), University of Oulu, Oulu, Finland, marko.sonkki@oulu.fi
}

\begin{abstract}
The article proposes a novel design for a high gain dual-band millimeter wave (mm-wave) antenna at $\mathbf{2 6 . 5}$ and $36.5 \mathrm{GHz}$ frequency bands using flexible substrate and Coplanar waveguide (CPW) technology. The CPW is able to provide $1.2 \mathrm{GHz}$ bandwidth at $26.5 \mathrm{GHz}$ center frequency and $3 \mathrm{GHz}$ bandwidth at $36.5 \mathrm{GHz}$ with 7.2 and $9.7 \mathrm{dBi}$ gain, respectively. The antenna is composed of a square patch and L-shape slots that are placed symmetrically with distances less than $\lambda / 4$ (upper band) and the antenna has total dimensions of $16 \mathrm{~mm} \times 19 \mathrm{~mm}$. The simulated antenna results are presented in terms of radiation patterns and return loss, and maximum gain as a function of frequency. The performance at millimeter wave and structure flexibility capability are demonstrated that the antenna has potential to implement in the upcoming 5G communication networks.
\end{abstract}

Index Terms-Dual band, felexible substate, inject printing, mm-wave antenna, Polyethylene terephthalate.

\section{INTRODUCTION}

Clients demand to access high speed data in mobile phone is the main motivation for telecommunication network that they are immigrating toward high bandwidth wireless communication 5th generation (or 5G) networks. Regarding huge growing demand to access wireless communication, there is challenging problem such energy consumption. Therefore, the upcoming $5 \mathrm{G}$ wireless systems have to comply three main requirements: having a high throughput, simultaneously serving many users, and having less energy consumption [1].

Certainly, the mm-wave technology has some advantages (higher accuracy, miniature devices, and less energy consumption, and wider available bandwidth) and drawbacks (Vulnerability, higher sensitive). Also, there is another complex difficulty to integrate tiny mm-wave devices.

In the upcoming $5 \mathrm{G}$ network, the antenna is the key component of the transceiver in wireless communication systems. In fact, as the last (first) block of the transceiver, its performance has a major influence on the system performance. Specifically, from energy efficiency perspective the antenna has a direct impact on the overall energy efficiency of the wireless communication system.

The mmw communication networks have been successfully applied to utilize in the indoor scenarios, but there is still several barrier to apply for the outdoor scenario
[2] including: high attenuation path loss (because of medium absorption ), sensitive to blockage by obstacle. To overcome the blockage, the dual-band antenna can provide two high gain patterns in different frequency, particularly on non-line of sight (NLOS) scenarios. Consequently, several tackling methods have been reported including: implementing array antenna, and beamforming, reasonably $[3,4]$.

To operate at mm-wave frequency, the short-range wireless communication architecture is recommended that requires the high gain antennas [2]. As it is known the array antenna is able to increase the directivity and reduce the beam width of the antenna which reduces signal interference and that may also reduce the energy consumption.

To design array antenna, patch antenna is the center of attention, regarding their advantages (planar structure and compatibility to mount on non-uniform surfaces). The printable substrate is using to design patch antenna with two technologies including: microstrip and CPW technology. Recent progress to improve the flexibility of patch antennas provide robust, efficient, and cost-effective methods of fabrication even mass-production and reduce the cost of antenna. Printed circuit technology has aimed as a promising solution to fabricate flexible patch antenna for low-cost mass production [5].

To fabricate (or print) the flexible substrate material, inkjet printing is a significant candidate as a fast and precise method of antenna fabrication. Currently, several conducting inks are available in market for inkjet printers. Their performance to design mm-wave antennas already have been demonstrated and implemented [6].

Polyethylene terephthalate (PET) is a thermoplastic polymer and is a good candidate to implement as an antenna substrate. To design antenna using PET, already several research works have been done and reported successfully [79].

The inkjet-printed millimeter-wave antenna $(60 \mathrm{GHz})$ on Polyethylene Terephthalate (PET) substrate has been proposed using CPW technology [6]. Cavity perturbation method already has been implemented to characterize the PET substrate for $10 \mathrm{GHz}$ and above [10]. The PET has its intrinsic properties including: low cost, high flexibility, and resistive towards environmental effects. PET with different permittivity and loss-tangent are available. 
Several studies have been done to design dual band mm-wave antenna for the 5G application using two technologies including: microstrip [11-13] and CPW [14, 15]. To design a high-performance antenna, the tradeoff between gain-bandwidth is unavoidable.

To enhance two parameters (gain and $\mathrm{BW}$ ), not also circuit technique has been using and but also selecting appropriate substrate material are considering effectively (as a new trend in the research line to design antenna).

Recently, the low-cost flexible PET (NB-WF-3GF100 from Mitsubishi Paper Mills Ltd.) and coplanar wave guide (CPW) have been used to design mm-wave antenna for $5 \mathrm{G}$ application. It was able to cover Ka band (26.5-40 GHz) [16] with realized gain above $4 \mathrm{dBi}$.

To compensate the high path loss in $5 \mathrm{G}$ network, the high gain antenna is required in both transmitter and receivers. In this paper, the dual band mm-wave antenna is designed using CPW technology via single feed line by aim of the flexible PET substrate. It is able to perform in two frequency bands $26.5 / 36.5 \mathrm{GHz}$ with different bandwidths $1.2 \mathrm{GHz}$ and 3 $\mathrm{GHz}$ and with gains $7.5 \mathrm{dBi}$ and $9.7 \mathrm{dBi}$ respectively. In fact, the size geometry is contributed to achieve higher gain in two bands compared to previous research works. Beside it intrinsic advantages on flexible and low-cost substrate.

\section{ANTENNA DESIGN}

\section{A. Selecting Substrate and geometry characterization}

The antenna is designed using a CPW feed line (silver conductor), the substrate with dimension $16 \mathrm{~mm} \times 19 \mathrm{~mm}$ with relative dielectric constant $\varepsilon_{\mathrm{r}}=3.2$ and loss-tangent 0.0022 , and thickness of $135 \mu \mathrm{m}$. Symmetric L-slots are etched from the patched surface (left and right sides) which is illustrated in Fig. 1. The L-slots enable the antenna to operate at two frequency bands and its dimensions and geometry are optimized. The group L-slots are helped to achieve the high gain. They are placed with $2.3 \mathrm{~mm}$ apart (less than $\lambda / 4$ at upper-band) with $0.1 \mathrm{~mm}$ width. The antenna's dimension parameters details are provided in

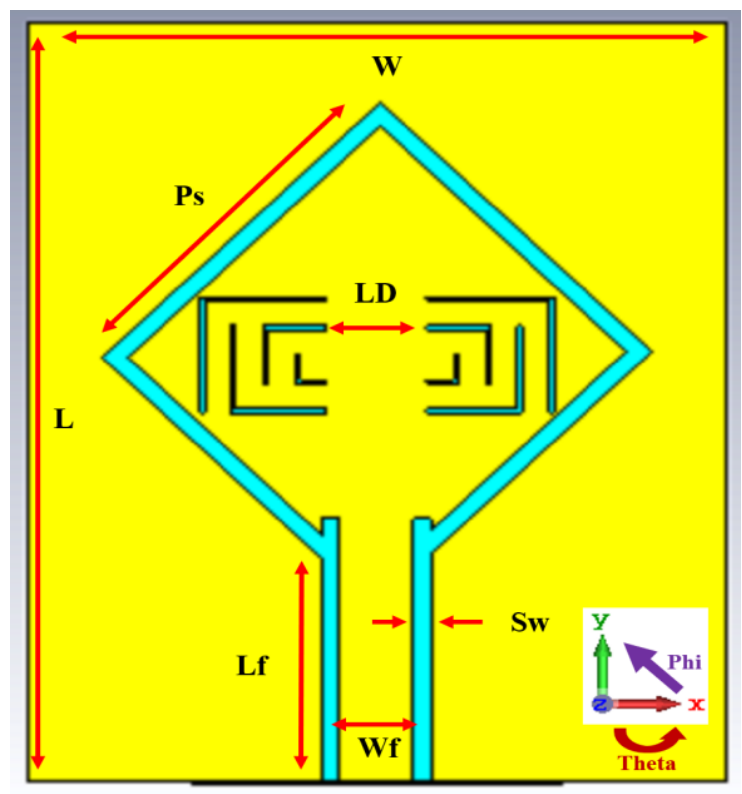

Fig. 1. Simulated rectangular CPW antenna using PET substrate.
Table 1.

TABLE I. OPTIMIZED DIMENSIONS.

\begin{tabular}{|c|c|}
\hline \multicolumn{2}{|c|}{ Antenna Dimension } \\
\hline parameters & Units $(\mathrm{mm})$ \\
\hline Wf & 1.7 \\
\hline Lf & 5.5 \\
\hline Ps & 7 \\
\hline Sw & 0.4 \\
\hline LD & 2.3 \\
\hline W & 16 \\
\hline L & 19 \\
\hline
\end{tabular}

\section{RESULTS AND DISCUSSION}

The antenna design is simulated by using CST Microwave Studio software. Its performance is optimized to achieve high gain at the desired dual-bands. The L-slots dimensions are optimized to achieve high gain in both bands.

\section{A. Antenna's Return Loss}

The return loss of the simulated antenna structure is presented in Fig. 2 in terms of $S_{11}$. The simulation results show that the antenna is able to cover two operating bands; $26.5 \mathrm{GHz}$ and $36.5 \mathrm{GHz}$ with $1.2 \mathrm{GHz}$ and $3 \mathrm{GHz}$ bandwidths, respectively.

\section{B. Gain and Radiation performance}

The antenna maximum gain is presented as a function of frequency in Fig. 3. The results show 7.2 and $9.7 \mathrm{dBi}$ gain at frequencies 26.5 and $36.5 \mathrm{GHz}$, respectively. The gain of antenna at the higher operating frequency (i.e. $36.5 \mathrm{GHz}$ ) brings an eye-catching benefit for the antenna from the propagation point of view: the higher the frequency of propagating wave, the more the path loss. Under this circumstance, to compensate the higher amount of path loss, it is preferred to have a high-gain antenna. The high gain of the designed antenna at its second band $(36.5 \mathrm{GHz})$ will be practical to mitigate the effect of higher path loss in the link

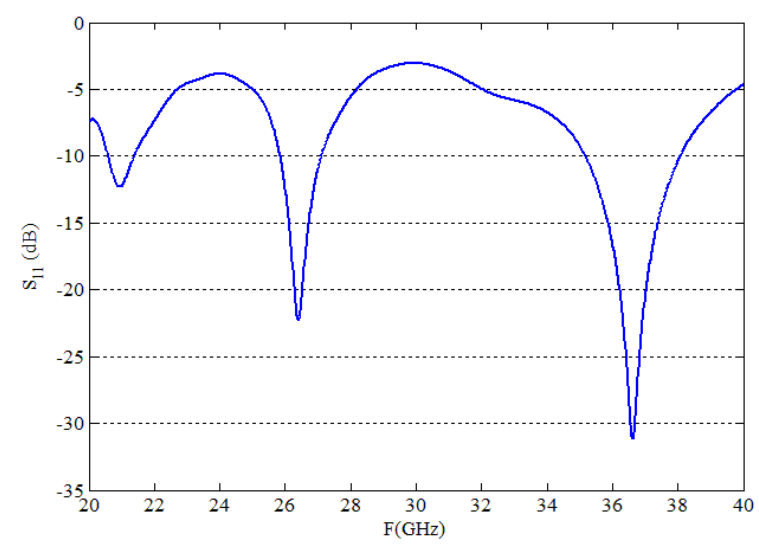

Fig. 2. Return Loss of the proposed antenna 


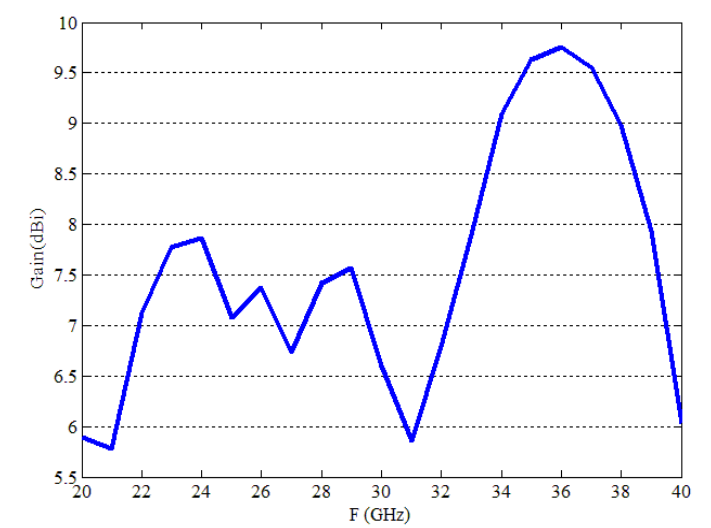

Fig. 3. High Gain Dual-Band Millimeter wave Antenna Using Flexible PET Substrate.

-budget. The antenna's pattern is presented on E-and H-plane (Fig. 4) for two center frequencies with $95 \%$ total efficiency.

\section{Surface Current performance}

The surface current distributions are shown in the Fig. 5 at the $26.5 \mathrm{GHz}$ and $36.5 \mathrm{GHz}$ center frequencies. As it is clear from the surface current in the figure, the current direction at the both studied frequencies are not generally changed and therefore, the antenna's radiating mode will not change at these frequencies.

\section{Review Study on Recent Dual-Band mm-wave Anennas}

This paper also presents a comparative study on recent published Dual-band mm-wave antenna on 5G network that shows the main challenge between bandwidth-gain still playing as some major restricting parameters. The antenna presented in this paper is able to achieve higher gain and higher bandwidth simultaneously compared to the previous studies [11, 12, 14].

The elliptical slot with semicircular and triangular sector patch antenna have been used to propose antenna using microstrip technology that was able radiate into two frequencies $30.5 / 41.5 \mathrm{GHz}$ with bandwidth $1.5 \mathrm{GHz}$ [12].

Another dual-band antenna has been proposed using sector-disk patch geometry and proximity-technique feed line. To enhance bandwidth, tow techniques has been utilized including: etching the elliptical shaped aperture on the ground plane and shunt stub. To avoid interferences, the notched bandwidth has been defined using $\pi$-shaped slot etch in the feed line [11].

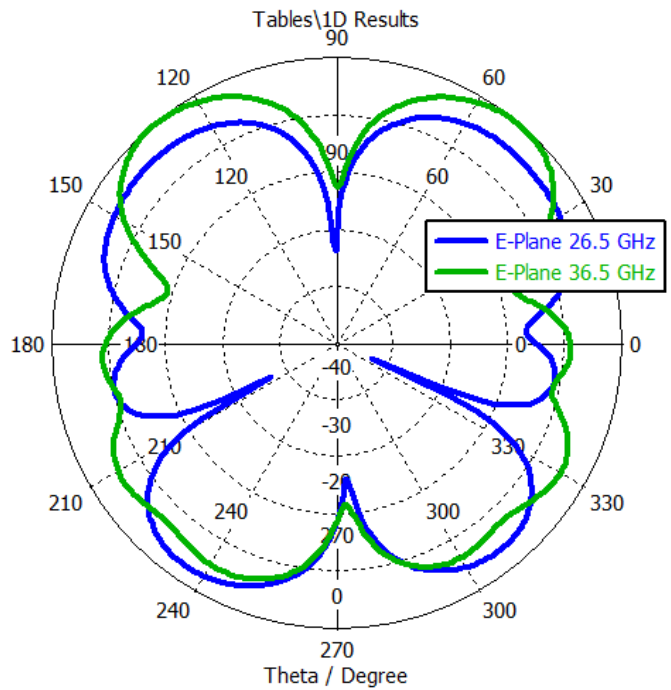

(a)

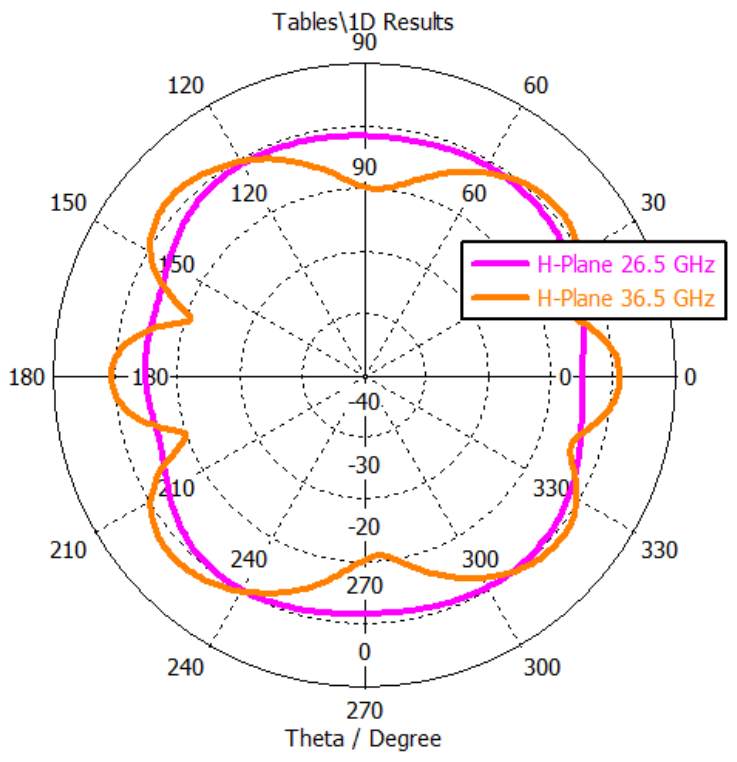

(b)

Fig. 4. Radiation pattern of the proposed antenna on, a) E_Plane ( $y z$ plane), b) H_plane ( $x z$ plane). 
TABLE II. REVIEW STUDY ON RECENT PUBLISHED WORK.

\begin{tabular}{|c|c|c|c|c|}
\hline Ref. & $\begin{array}{c}\text { Frequency } \\
(\boldsymbol{G H z})\end{array}$ & $\begin{array}{c}\text { Substrate } \\
\boldsymbol{\varepsilon}_{r}\end{array}$ & $\begin{array}{c}\text { Band Width } \\
(\mathbf{G H} z)\end{array}$ & $\begin{array}{c}\text { Gain } \\
(\boldsymbol{d B i})\end{array}$ \\
\hline$[11]$ & $28 / 38$ & 2.2 & 4 & 7 \\
\hline$[12]$ & $30.5 / 41.5$ & 3.2 & 1.5 & - \\
\hline$[14]$ & $28 / 38$ & 3.93 & 3.34 & 5.6 \\
\hline This work & $26.5 / 36.5$ & 3.2 & 3 & 9.7 \\
\hline
\end{tabular}

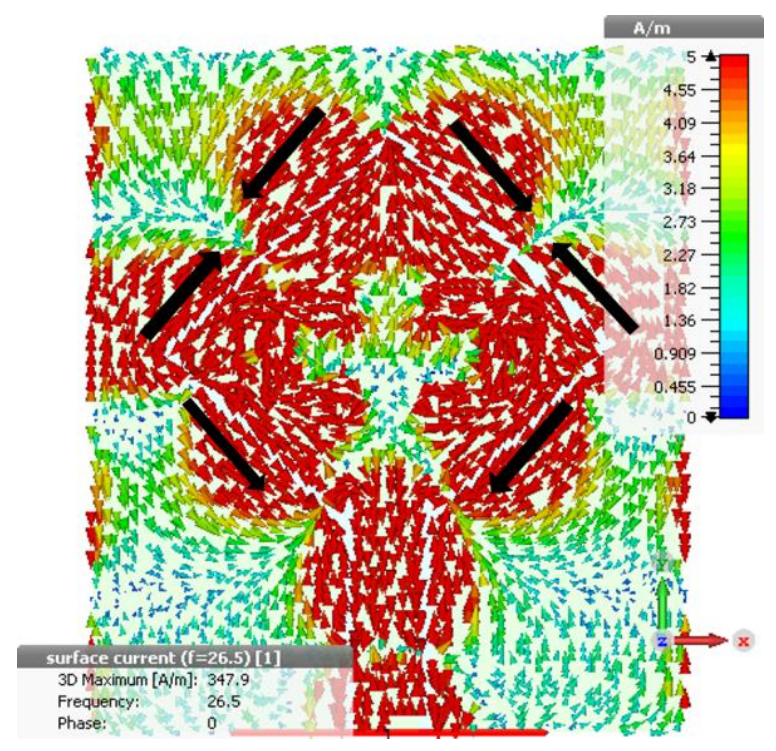

(a)

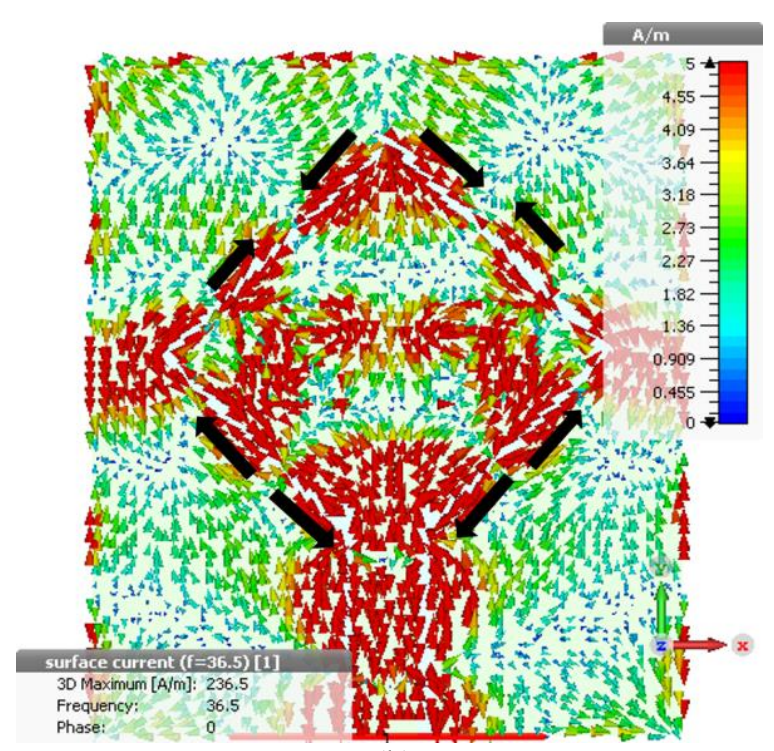

(b)

\section{CONCLUSION}

High gain dual-band antenna at 26.5 and $36.5 \mathrm{GHz}$ frequencies was designed using flexible and printable PET substrate on CPW technology. The designed antenna benefits from high gain and acceptable bandwidth at both operating frequencies, making it quite appropriate to be utilized in the upcoming $5 \mathrm{G}$ networks in which high gain antenna will be necessary at both transmitter and receiver side. The proposed antenna was simulated by commercial full-wave package CST MWS and the results show the proper behavior of the antenna.

\section{ACKNOWLEDGMENT}

Thanks to Iran's National Elites Foundation (INEF) for financially supporting this research work. Authors are also grateful to Iran Telecommunication Research Center (ITRC), Iran and University of Oulu, Finland for providing the research environments.

\section{REFERENCES}

[1] H. Q. Ngo, Massive MIMO: Fundamentals and system designs. Linköping University Electronic Press, 2015.

[2] J. Zhang, X. Ge, Q. Li, M. Guizani, and Y. Zhang, "5G Millimeter-Wave Antenna Array: Design and Challenges," IEEE Wireless Communications, vol. 24, no. 2, pp. 106-112, 2017.

[3] S. Kutty and D. Sen, "Beamforming for Millimeter Wave Communications: An Inclusive Survey," IEEE Communications Surveys \& Tutorials, vol. 18, no. 2, pp. 949-973, 2016.

[4] T. S. Rappaport et al., "Millimeter Wave Mobile Communications for 5G Cellular: It Will Work!," IEEE Access, vol. 1, pp. 335-349, 2013.

[5] S. R. Forrest, "The path to ubiquitous and low-cost organic electronic appliances on plastic," Nature, 10.1038/nature02498 vol. 428, no. 6986, pp. 911-918, 04/29/print 2004.

[6] K. Hettak, T. Ross, R. James, A. Momciu, and J. Wight, "Flexible polyethylene terephthalate-based inkjet printed CPWfed monopole antenna for $60 \mathrm{GHz}$ ISM applications," in Microwave Conference (EuMC), 2013 European, 2013, pp. 1447-1450: IEEE.

[7] L. Wang, Y.-X. Guo, B. Salam, and C. W. A. Lu, "A flexible modified dipole antenna printed on PET film," in Antennas and Propagation (APCAP), 2012 IEEE Asia-Pacific Conference on, 2012, pp. 239-240: IEEE.

[8] N. J. Kirsch, N. A. Vacirca, E. E. Plowman, T. P. Kurzweg, A. K. Fontecchio, and K. R. Dandekar, "Optically transparent conductive polymer RFID meandering dipole antenna," in RFID, 2009 IEEE International Conference on, 2009, pp. 278 282: IEEE.

[9] H. K. Yoon, W. S. Kang, Y. J. Yoon, and C.-H. Lee, "A CPWfed flexible monopole antenna for UWB systems," in Antennas and Propagation Society International Symposium, 2007 IEEE, 2007, pp. 701-704: IEEE.

[10] S.-H. Chao, "Measurements of microwave conductivity and dielectric constant by the cavity perturbation method and their errors," IEEE transactions on microwave theory and techniques, vol. 33, no. 6, pp. 519-526, 1985.

[11] O. M. Haraz and M. M. M. Ali, "A millimeter-wave circular reflectarray antenna for future 5G cellular networks," in 2015 IEEE International Symposium on Antennas and Propagation \& USNC/URSI National Radio Science Meeting, 2015, pp. 15341535 . 
[12] N. Kathuria and S. Vashisht, "Dual-band printed slot antenna for the $5 \mathrm{G}$ wireless communication network," in Wireless Communications, Signal Processing and Networking (WiSPNET), International Conference on, 2016, pp. 1815-1817: IEEE.

[13] H. Aliakbari, A. Abdipour, R. Mirzavand, A. Costanzo, and P. Mousavi, "A single feed dual-band circularly polarized millimeter-wave antenna for 5G communication," in 2016 10th European Conference on Antennas and Propagation (EuCAP), 2016, pp. 1-5.

[14] W. Ahmad and W. T. Khan, "Small form factor dual band (28/38 GHz) PIFA antenna for 5G applications," in Microwaves for Intelligent Mobility (ICMIM), 2017 IEEE MTT-S International Conference on, 2017, pp. 21-24: IEEE.

[15] M. Koohestani, A. A. Moreira, and A. K. Skrivervik, "A novel compact CPW-fed polarization diversity ultrawideband antenna," IEEE Antennas and Wireless Propagation Letters, vol. 13, pp. 563-566, 2014.

[16] S. F. Jilani and A. Alomainy, "Planar millimeter-wave antenna on low-cost flexible PET substrate for 5G applications," in Antennas and Propagation (EuCAP), 2016 10th European Conference on, 2016, pp. 1-3: IEEE. 